\title{
SYNTHESIS OF FUNCTIONALIZED PROPYLAMINEPOLYANILINES AND INVESTIGATION OF THEIR PROPERTIES
}

\author{
M.A. TAKASSI ${ }^{a}$, AND A. ZADEHNAZARI*,a \\ ${ }^{a}$ The department of Science, Petroleum University of Technology, Ahwaz, Iran
}

\begin{abstract}
In present investigation, N-substituted propylaminepolyaniline ( $N$-PAPANI) was prepared by reaction of dimethylsulfoxide solution of polyaniline (emeraldine) base with sodium hydride, then reacted with 3-chloropropylaminehydrochloride. The $N$-PAPANI was characterized using reflectance Fourier transformation infrared (FTIR), cyclic voltammetry, UV-vis spectroscopy and chemical analysis. Polyaniline (PANI) was also subjected to a reaction with 3-chloropropylaminehydrochloride and ferric chloride in acetonitrile as solvent under refluxing temperature $\left(85-90^{\circ} \mathrm{C}\right)$ (Friedel-Craft synthetic method) to produce ring substituted functionalized PAPANI. Characterization of PAPANI was carried out using various aforementioned techniques. PANI and $N$-PAPANI polymers exhibited strong UV-vis absorption maxima at 273-294 nm in solution. Cyclic voltammograms of the polymers on an indium-tin oxide (ITO)-coated glass substrate exhibited one pair of reversible redox couples at half-wave oxidation potentials $\left(E_{1 / 2}\right)$ around $0.84-0.86 \mathrm{~V}$ and $0.88 \mathrm{~V}$, respectively, versus $\mathrm{Ag} / \mathrm{AgCl}$ in an acetonitrile solution. The obtained polymers showed good solubility in common organic solvents such as tetrahydrofuran and chloroform compared with emeraldine which is difficult to process due to lack of solubility. Furthermore, these polymers are air stable.
\end{abstract}

Key words: N-propylaminepolyaniline; direct oxidation, Friedel-Craft synthesis

\section{INTRODUCTION}

Finding of conducting polymers is one of the most electrifying phenomena occurred in material science in the past four decades $[1,2]$. Various potential electrochemical applications have been projected and reported [3-5]. The problem for use of conducting polymers is the low process ability of these materials. To diminish this problem, one of the approaches is taken in the past twenty years, to produce substituted conducting polymers. The substituted polymer can regularly be generated using oxidative polymerization of the monomer. But, in many circumstances, the anticipated substituted monomer, is either too hard to oxidize or sensitive to oxidative and acidic environments. Innovative synthetic approaches are required [6,7]. A plan would be to use a monomer containing a reactive substituent group to make a precursor polymer, and then it can be altered to form the anticipated structure [8]. Another plan would be to make the polymer, and then try to add or substitute the substituent with favorite functional group to the backbone of the polymer $[9,10]$.

To synthesize conducting polymers for particular jobs it is essential to develop the procedures to present a wide-ranging of functional groups. A simple technique is to polymerize substituted monomer bearing functional groups of interest. But, in many circumstances the favorite moiety is either too hard to oxidize using common oxidative agents or too sensitive to oxidative or acidic environments. Polyaniline (PANI) like other conducting polymers is hard to process due to poor solubility in most ordinary solvents. The addition of useful groups as substituent on the PANI backbone can yield materials which are more soluble and thus more readily processible, while still retaining the physical characteristic of PANI [11]. Making of substituted PANI appears to be straight forward and it can be produced by oxidative polymerization of corresponding monomer. Nevertheless, in many cases the desired functional group is either too hard to oxidize using common oxidative reagents or too sensitive to oxidative or acidic environments. Many different synthetic approaches have been described to synthesize substituted PANI [12-14]. Many ring substituted PANI and N-substituted PANI have been reported [15-18], but no report was made on the successful preparation of N-alkyl amine substituted PANI.

Herein, we report a strategy for synthesizing two types of substituted PANIs using nucleophilic displacement and Friedel-Craft method. First, N-propylamine substituted PANI was synthesized by reaction of 3-chloropropylamine and sodium hydride with dimethylsulfoxide (DMSO) solution of PANI; then, the halogen containing substituent reacted with polymer in the presence of ferric chloride $\left(\mathrm{FeCl}_{3}\right.$ ) (a Lewis acid) in acetonitrile as solvent under reflux to make the anticipated ring substituted PANI. The 3-chloropropylamine ring substitution of PANI was carried out on a conducting glass and also on the surface of a glassy carbon electrode for electrochemical analysis.

\section{EXPERIMENTAL SECTION}

Material and reagents

3-chloropropylaminehydrochloride, aniline, ammonium persulfate, acetonitrile, DMSO, tetrahydrofurane (THF) and sodium hydride were purchased from Aldrich Chemical Co. (Milwaukee, WI). Hydrochloric acid was purchased from Fisher Scientific (Pittsburgh, United States).

Instrumental setup

The glassy carbon (GC) electrodes (3 mm diameter) were purchased from Bioanalytical Science. Cyclic voltammetry was performed with a BAS (Bioanalytical System) potentiostat (Model 100) (West Lafayette, United States). In the voltammetric experiments, a three-electrode configuration was used, including $\mathrm{Ag} / \mathrm{AgCl}$ reference electrode, indium-tin oxide (ITO) glass slide with polymer as working electrode and a platinum wire counter electrode. The reflectance FTIR spectra of polymer was obtained using Nicolet NEXUS 870 FTIR instrument (Minnesota, United States). The polymer was treated with $1 \mathrm{M}$ aqueous $\mathrm{NaOH}$ solution to remove the doped $\mathrm{HCl}$ from the polymer. The polymer was washed with water, and then, it was dried under continuous vacuum for 24 hours. The FTIR reflectance spectrum of N-PAPANI was obtained and compared with the FTIR reflectance spectrum of emeraldine base. The uv-vis spectrum was obtained using an Agilent 8453 spectrophotometer (San Diego, CA, United States).

\section{Reaction of 3-chloropropylamine with PANI}

The emeraldine base form of PANI (Scheme 1) was prepared by means of chemical oxidation method described by MacDiarmid [19], an aqueous solution of ammonium persulfate was added to a solution of aniline dissolved in $1 \mathrm{M}$ aqueous $\mathrm{HCl}$ at about $5^{\circ} \mathrm{C}$.s The proportion of aniline and ammonium peroxide was 1:1, and after 1 hour the precipitate that had formed was removed by filtration, then it was washed with $1 \mathrm{M} \mathrm{HCl}$ and dried under continuous vacuum for about 48 hours. The resulting emeraldine form of PANI (Scheme 1) was extracted with THF until the extract was colorless. The dried PANI $(0.25 \mathrm{~g})$ was reacted with excess $\mathrm{NaH}(0.4 \mathrm{~g})$ in anhydrous dimethylsulfoxide solution at $45{ }^{\circ} \mathrm{C}$ under dried nitrogen atmosphere for 6 hours to give a black solution. The black solution was subsequently reacted with excess 3-chloropropylaminehydrochloride $(0.4 \mathrm{~g})$ at a temperature of $81^{\circ} \mathrm{C}$ for 24 hours. The resulting solution was precipitated with $1 \mathrm{M} \mathrm{HCl}$ aqueous solution. A blue to black precipitate was formed, and the precipitate was washed with $\mathrm{HCl}$ then with acetonitrile. The precipitate was dried under continuous vacuum for 24 hours; N- propylaminepolyaniline (N-PAPANI), was formed.

The $\mathrm{HCl}$ doped PAPANI was sent for chemical analysis to Guelph Chemical Laboratories LTD (Guelph, Ontario, Canada). The result of Chemical analysis suggests that substitution occurred on all secondary amine groups in the polymer (Scheme 1).

Reaction of 3-chloropropylaminehydrochloride and ferric chloride with PANI deposited on the GC electrode

The oxidative polymerization of aniline was carried out to produce PANI, when $40 \mathrm{mM}$ of aniline was dissolved in $25 \mathrm{~mL} 0.5 \mathrm{M}$ aqueous hydrochloric acid solution. The potential of the GC electrode was scanned between -0.4 to $1.1 \mathrm{~V}$ vs. $\mathrm{Ag} / \mathrm{AgCl}$ at a scan rate $100 \mathrm{mV} / \mathrm{s}$, the polymerization was stopped at $-0.4 \mathrm{~V}$ when the charge passed from the reduction of deposited polymer reached to $0.65 \mathrm{mC}$. The PANI film had a green color. The film was washed with distilled water, then with tetrahydrofurane (THF). The GC electrode with film 
was immersed in a large glass tube with THF solution contained $0.01 \mathrm{~g} \mathrm{FeCl}_{3}$ and $0.5 \mathrm{~mL}$ of 3-chloropropylamine, and the top of tube was closed to prevent evaporation of the solvent. The reaction was carried out at a temperature of $60{ }^{\circ} \mathrm{C}$ for 24 hours in a temperature controlled mineral oil bath. The cyclic voltammogram of the film after reaction is similar to those 1,2,4-trisubstituted PANI reported by Freund [20].<smiles>CC(C)(C)N=C1C=CC(=Nc2ccc(Nc3ccc(Nc4ccc(C(C)(C)C)cc4)cc3)cc2)C=C1</smiles>

PANI

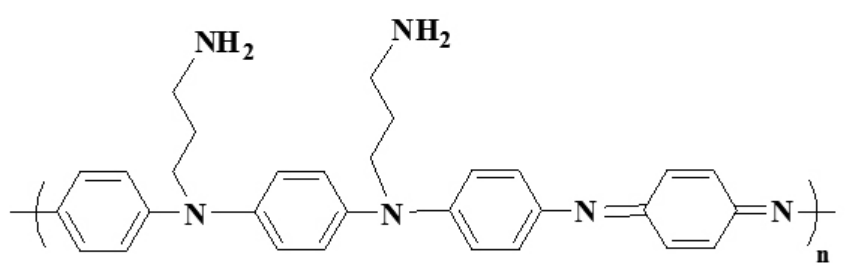

\section{N-PAPANI}

Scheme 1. Chemical structure of PANI and N-PAPANI. PANI

Reaction of 3-chloropropylaminehydrochloride and ferric chloride with

The emeraldine base form of PANI was prepared using the above mentioned procedure. The PANI was extracted with THF until the extract was colorless. The dried PANI $(0.25 \mathrm{~g})$ reacted with 3-chloropropylaminehydrochloride $(0.5$ $\mathrm{mL})$ and ferric Chloride $(0.01 \mathrm{~g})$ in anhydrous dimethylsulfoide solution at $81^{\circ} \mathrm{C}$ for 24 hours. The black solution, $o$-PAPANI was precipitated with 1 $\mathrm{M}$ aqueous $\mathrm{HCl}$ solution, a dark green to black precipitate was formed the filtrate was greenish blue; the precipitate was washed with $1 \mathrm{M} \mathrm{HCl}$, then with acetonitrile. The precipitate $o$-PAPANI was dried under continuous vacuum for 48 hours.

The $\mathrm{HCl}$ doped PAPANI was sent for chemical analysis to Guelph Chemical Laboratories LTD (Guelph, Ontario, Canada). The result of Chemical analysis suggests that substitution occurred on all benzenoid rings in the polymer (Scheme 2).

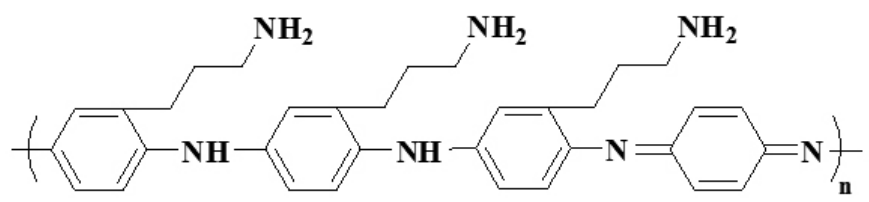

$o$-PAPANI

Scheme 2. Chemical structure of $o$-PAPANI.

\section{RESUSLTS AND DISCUSSION}

In this study, PANI (emeraldine) base was reacted with sodium hydride in DMSO solution under nitrogen atmosphere. In this reaction the emeraldine base was probably converted to an emeraldine base without $\mathrm{H}$ on nitrogen (anionic nitrogen) [21]. The solution was subsequently reacted with excess 3-chloropropylaminehydrochloride to obtain $\mathrm{N}$ - propylaminepolyaniline (N-PAPANI) (Scheme 1).

The reflectance FTIR spectrum of N-PAPANI, Figure 1, showed that the ratio of absorption intensity at $1594 \mathrm{~cm}^{-1}$ due to the quinoid ring to that at $1506 \mathrm{~cm}^{-1}$ due to the benzenoid ring is $1: 3[22,23]$. The presence of weak to medium, C-N aliphatic absorptions at $1020 \mathrm{~cm}^{-1}$ and $1249 \mathrm{~cm}^{-1}$ due to primary aliphatic amine [24] and also the presence of an another absorption peak at $1265 \mathrm{~cm}^{-1}$ characteristic of aliphatic C-N which is attached to the polymer [25], all together confirm the N-substitution of propylamine on the PANI. The presence of an absorption peak at $831 \mathrm{~cm}^{-1}$, as it is in emeraldine base, Figure 2, characteristic of the $\mathrm{C}-\mathrm{H}$ out of plain bending vibrations of 1,4-disubstituted benzene ring [21] indicates that no ring substitution occurs in the present PANI derivative.

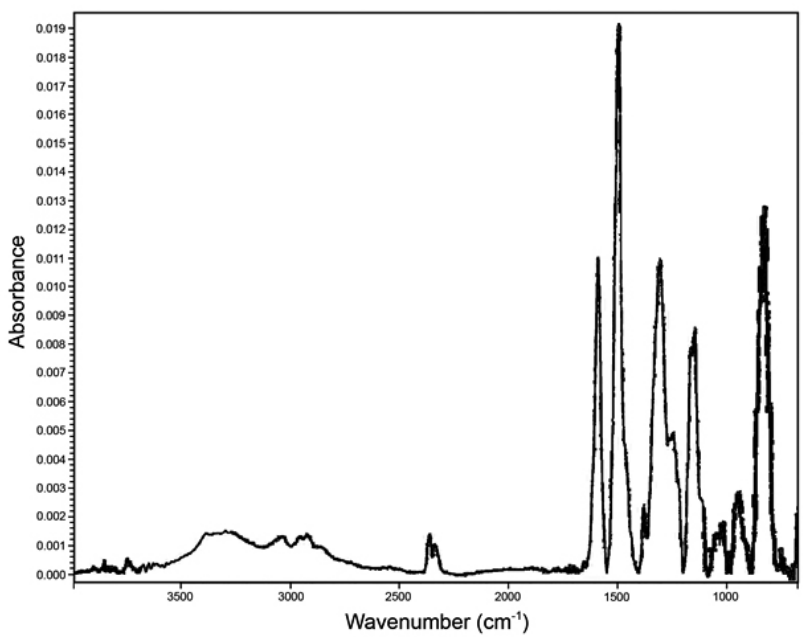

Figure 1. FTIR spectrum of N-PAPANI, compound 1.

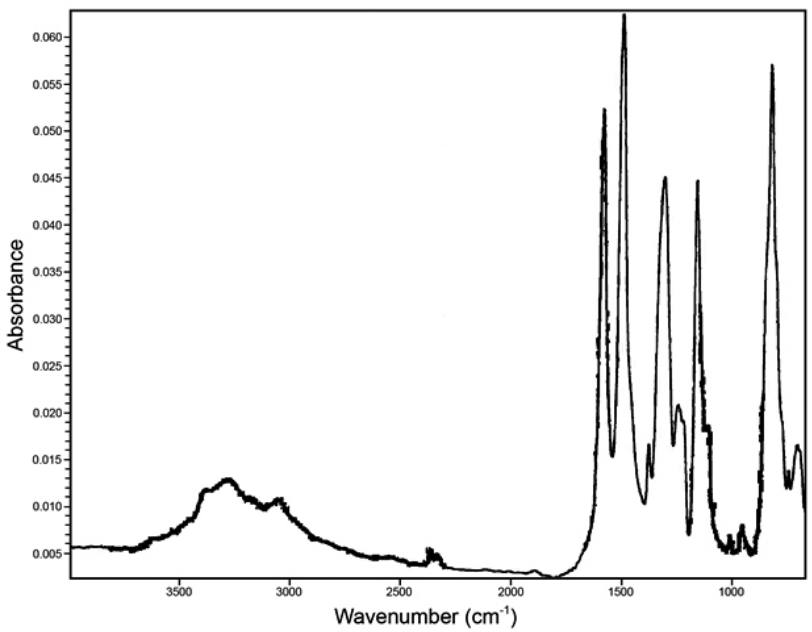

Figure 2. FTIR spectrum of PANI (emeraldine).

The redox behavior was observed by cyclic voltammetry conducted for the solid polymers on an ITO-coated glass substrate as working electrode in dry acetonitrile under nitrogen atmosphere. The cyclic voltammogram of bulk chemically prepared PANI and N-PAPANI are shown in Figures 3. A pair of reversible redox couple could be observed on the $\mathrm{CV}$ scans of two polymers. The PANI exhibited half-wave potential $\left(E_{1}\right)$ value of $0.84-0.86 \mathrm{~V}$ (Figures 3 , top) $\left(\mathrm{E}_{\text {onset }}=0.38 \mathrm{~V}\right)$ in the oxidative scan. The color of the polymer changed from green to blue because of electrochemical oxidation of the polymer. The oxidative and electrochromic reversibility is maintained on repeated scanning between 0 and $1.0 \mathrm{~V}$ (vs. $\mathrm{Ag} / \mathrm{AgCl}$ ). For N-PAPANI (Figures 3, bottom), $E_{1 / 2}$ value was $0.88 \mathrm{~V}$. The oxidative and electrochromic reversibility is maintained on repeated scanning between 0 and $1.1 \mathrm{~V}$. This result confirms that $\mathrm{N}$-substitution of the PANI amine group leads considerable stability to the radical cation species.

The chemical analysis of $\mathrm{HCl}$ doped N-PAPANI suggests that substitution occurred on all nitrogen atoms as it is shown in Scheme 1. The obtained results from the chemical analysis are given in Table 1. 


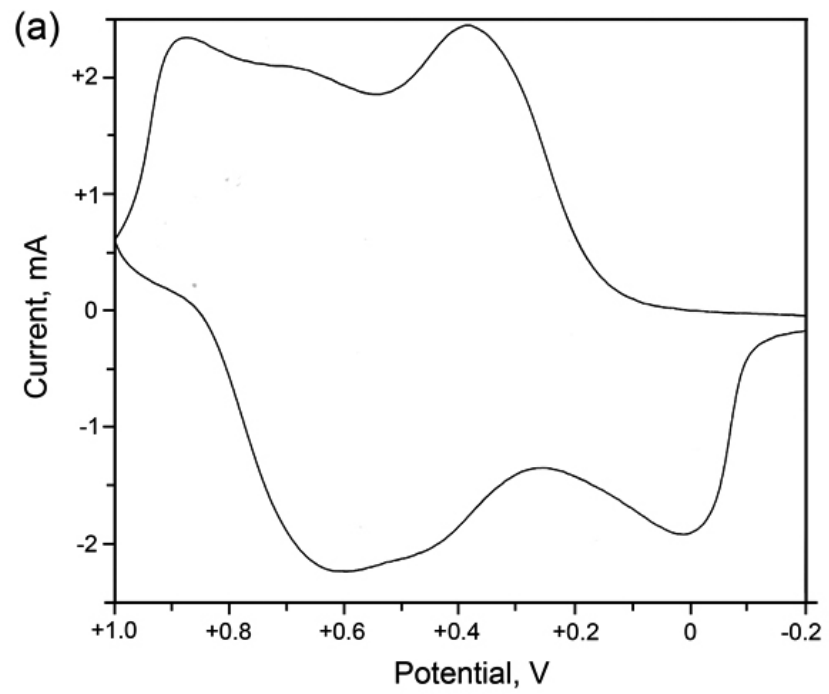

(b)

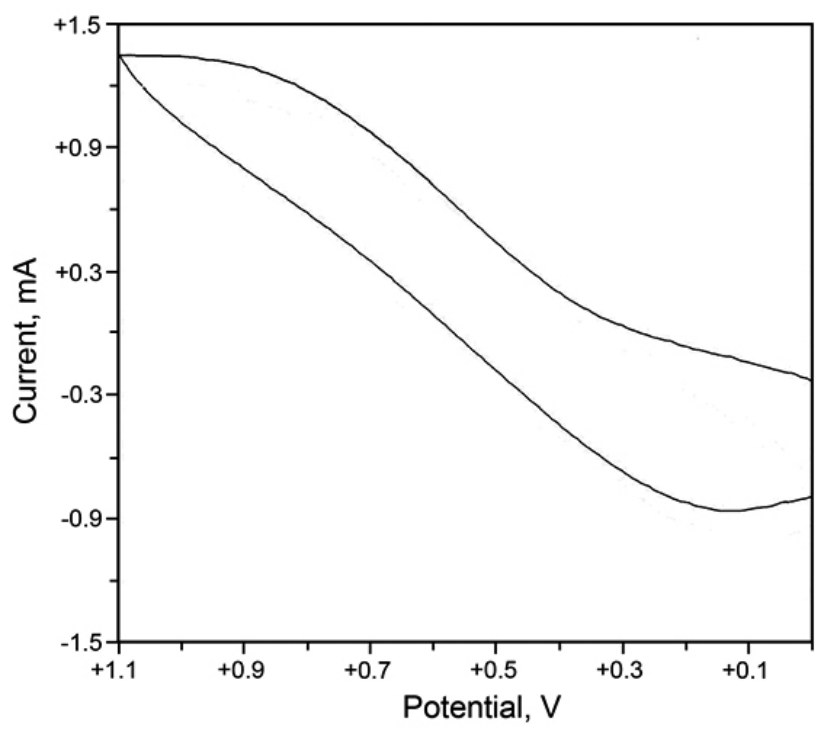

Figure 3. Cyclic voltammograms of (a) PANI and (b) N-PAPANI.

The UV-vis spectrum of DMSO solution of N-PAPANI (Figure 4) demonstrates a partial oxidation as a broad band at $600 \mathrm{~nm}$.

Table 1: The results of chemical analysis of N-PAPANI.

\begin{tabular}{|c|c|c|c|}
\hline N-PAPANI & $\%$ carbon & $\%$ hydrogen & $\%$ nitrogen \\
\hline Theoretical values & 75.6 & 6.7 & 17.6 \\
\hline Observed values ${ }^{a}$ & 53.7 & 4.9 & 8.9 \\
\hline $\begin{array}{l}\text { Standard PANI } \\
\text { (emeraldine) }\end{array}$ & $\%$ carbon & $\%$ hydrogen & $\%$ nitrogen \\
\hline Theoretical values & 79.6 & 5.0 & 15.4 \\
\hline Observed values ${ }^{a}$ & 74.1 & 4.7 & 14.4 \\
\hline
\end{tabular}

The Friedel-Craft synthetic method was also used to prepare PAPANI. PANI was produced by both direct oxidation and electrochemical oxidation of aniline on GC electrode.

The reflectance FTIR spectrum of PAPANI (Figure 5) showed that the ratio of absorption intensity at $1594 \mathrm{~cm}^{-1}$ due to the quinoid ring to that at $1506 \mathrm{~cm}^{-1}$ due to the benzenoid ring is 1:3 [23]. The absence of an absorption peak at $831 \mathrm{~cm}^{-1}$, as it is in emeraldine base, (Figure 2), characteristic of the
C-H out of plain bending vibrations of 1,4-disubstituted benzene ring [23] with the presence of splitting of this peak into two peaks at $823 \mathrm{~cm}^{-1}$ and $944 \mathrm{~cm}^{-1}$ indicative of 1,2,4-trisubstiution of benzene rings in the PANI. The presence of C-N aliphatic stretch absorption peaks at $1020 \mathrm{~cm}^{-1}$ and $1261 \mathrm{~cm}^{-1} \mathrm{due}$ to primary aliphatic amine, aliphatic C-H stretch at $2947 \mathrm{~cm}^{-1}, 2915 \mathrm{~cm}^{-1}$ and symmetrical stretching $v \quad \mathrm{CH}_{2}$ at $2847 \mathrm{~cm}^{-1}$ [23] confirms the propylamine substitution of PANI.

Last Blank spectrum

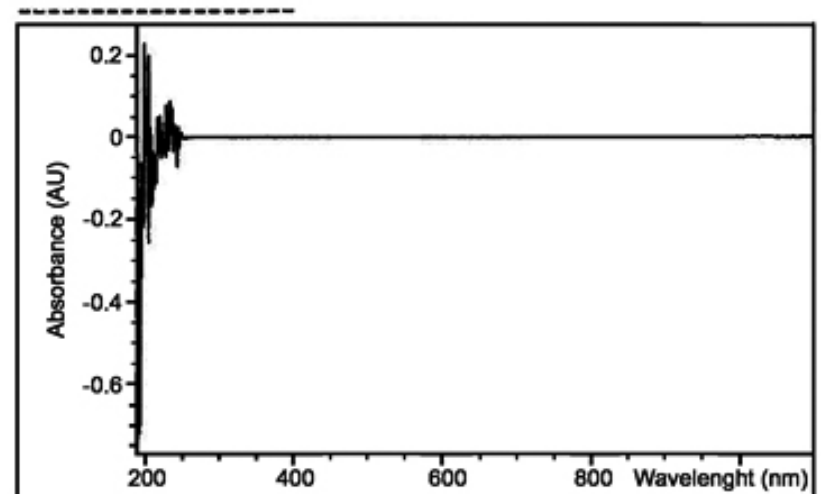

Overlaid Sample spectra

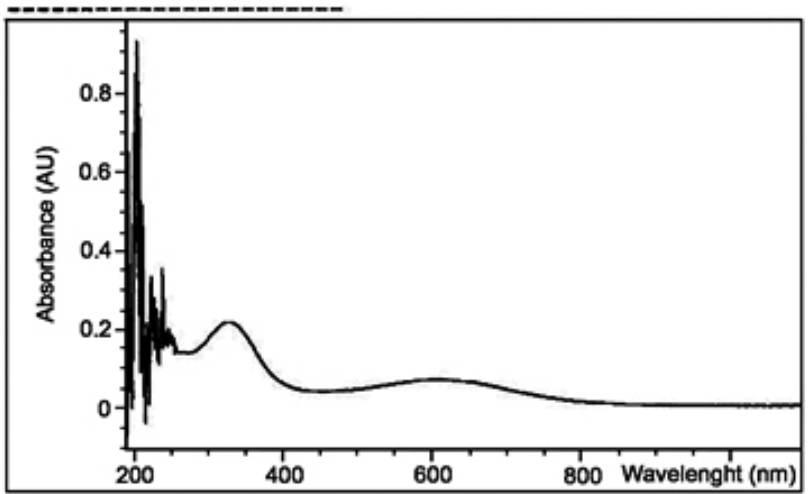

Figure 4. Uv-vis spectra of N-PAPANI and the solvent (blank).

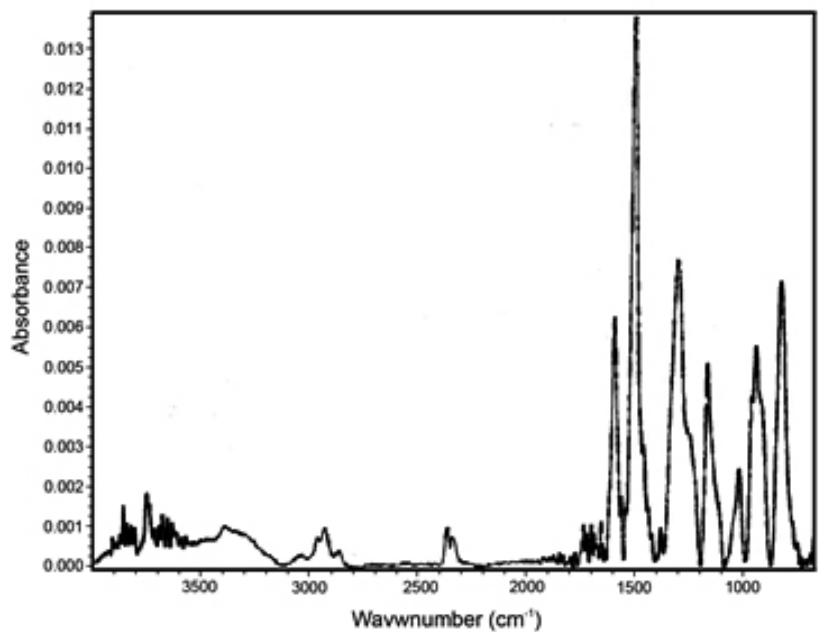

Figure 5. FTIR spectrum of $o$-PAPANI.

The cyclic voltammograms of electrochemically prepared PANI after its reaction with 3-chloropropylamine and ferric chloride is shown in Figure 6. The changes in voltammogram is a clear indication of 1,2,4-trisubstitution of benzene ring in PANI [20]. 


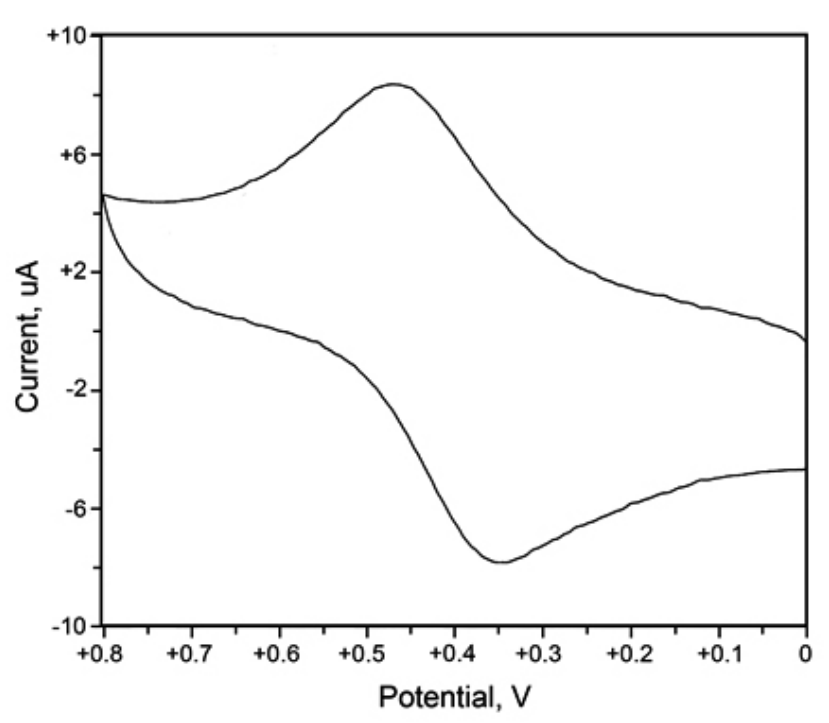

Figure 6. Cyclicvoltammogram of electrochemically prepared O-PAPANI.

The chemical analysis of $\mathrm{HCl}$ doped PAPANI perfectly suggests that substitution occurred on all benzenoid rings in the polymer. Three out of four rings in PANI were substituted, it makes the degree of substitution $75 \%$, while the degree of substitution for electrochemically prepared ring substituted dialkylamine is $25 \%$. Electrophilic substitution of 3-chloropropylaminehydrochloride in the presence of ferric chloride makes it possible to prepare any type of alkyl, alkylamine, alkylthiol [10], and other substitution on conducting polymers with a good degree of substitution.

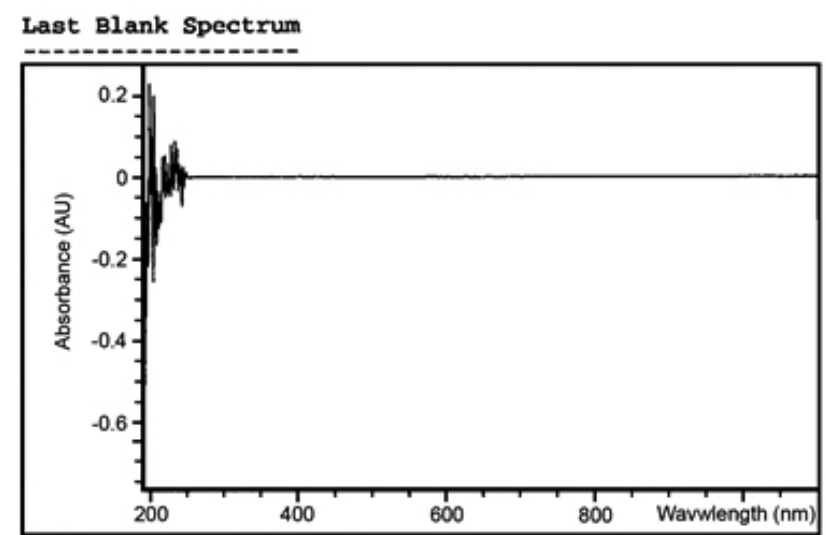

Overlaid Sample Spectra

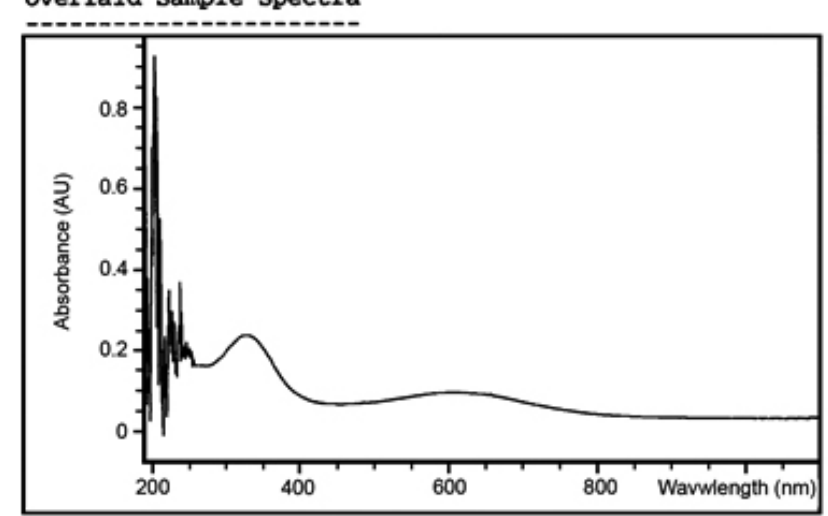

Figure 7. Uv-vis spectra of $o$-PAPANI and N-PAPANI.

\section{CONCLUSIONS}

Reaction of DMSO solution of PANI (emeraldine) base with sodium hydride to produce a material with the propylamine group bonded to the PANI backbone. The substitution reaction of polymer with 3-chloropropylamine and ferric chloride was also occurred via Friedel-Craft procedure. Although for bulk chemically prepared polymer this method is restricted due to low to moderate solubility of polymer in the proper solvent, but this technique works perfectly for polymers prepared on the surface of electrode or prepared on the surface of conducting glass slide. We suggest Friedel-Craft technique for synthesis is a good approach to overcome the above mentioned problems for preparation of substituted conducting polymers. The prepared air stable polymers were soluble in organic solvents such tetrahydrofuran and chloroform.

\section{ACKNOWLEDGEMENT}

We would like to thank Professor Michael S. Freund of university of Manitoba, Canada, for his generous support to this work. We would also like to thank Dr. Bhavana A. Deore and Carmen Recksiedler for their technical support.

\section{REFERENCES}

1. A.T. Lawal, G.G. Wallace, Talanta $119,133,(2014)$

2. U. Lange, V.M. Mirsky, Anal. Chim. Acta 687, 105, (2011)

3. M. Ates, Mater. Sci. Eng. C 33, 1853, (2013)

4. A. Ramanavicius, Y. Oztekin, Z. Balevicius, A. Kausaite-Mikstimiene, V. Krikstolaityte, I. Baleviciute, V. Ratautaite, A. Ramanaviciene, Procedia Eng. 47, 825, (2012)

5. N.K. Guimard, N. Gomez, C.E. Schmidt, Prog. Polym. Sci. 32, 921, (2007)

6. J. Alam, L.A. Dass, M.S. Alhoshan, A.W. Mohammad, Adv. Polym. Technol. 32, E189, (2013)

7. A.J. Heeger, Angew. Chem. Int. Ed 40, 2591, (2001)

8. C.O. Sanchez, A. Isla, C. Bustos, F. Diaz, N. Gatica, J. Chil. Chem. Soc. $55,233,(2010)$

9. E. Ortega, F. Armijo, I. Jessop, M.A. Del Valle, F.R. Diaz, J. Chil. Chem. Soc. 58, 1959, (2013)

10. M.A. Takassi, M.R. Shishesaz, Polym. Res. J. 7, 129, (2013)

11. J. Longun, J.O. Iroh, J. Appl. Polym. Sci. 128, 1425, (2013)

12. A.G. Yavuz, A. Uygun, H. Kaplan Can, Carbohydr. Res. 346, 2063, (2011)

13. H. Hasbullah, S. Kumbharkar, A.F. Ismail, K. Li, J. Membr. Sci. 397-398, $38,(2012)$

14. M.K. Kim, K. Shanmuga Sundaram, G. Anantha Iyengar, K.-P. Lee, Chem. Eng. J. 267, 51, (2015)

15. C. Li, S. Mu, Synth. Met. 144, 143, (2004)

16. J. Wang, D. Zhang, Adv. Polym. Technol. 32, E323, (2013)

17. S.D. Shinde, M. Jayakannan, J. Appl. Polym. Sci. 127, 1781, (2013)

18. E.-M. Kim, C.-K. Jung, E.-Y. Choi, C. Gao, S.-W. Kim, S.-H. Lee, O.P. Kwon, Polymer 52, 4451, (2011)

19. J.-C. Chiang, A.G. MacDiarmid, Synth. Met. 13, 193, (1986)

20. E. Shoji, M.S. Freund, Langmuir 17, 7183, (2001)

21. S.-A. Chen, G.-W. Hwang, J. Am. Chem. Soc. 116, 7939, (1994)

22. F. Wudl, R.O. Angus, F.L. Lu, P.M. Allemand, D. Vachon, M. Nowak, Z.X. Liu, H. Schaffer, A.J. Heeger, J. Am. Chem. Soc. 109, 3677, (1987)

23. H. Tang, A. Kitani, T. Yamashita, S. Ito, Synth. Met. 96, 43, (1998)

24. R.M. Silverstein, G.C. Bassler, T.C. Morrill Spectrometric Identification of Organic Compounds, 4th Ed. John Wiley \& Sons, 1981; pp. 128.

25. K. Nakanishi, P.H. Solomon Infrared Absorption Spectroscopy, 2nd ed.; Nankodo, Tokyo, 1977; pp. 33. 